Groups Geom. Dyn. 7 (2013), 109-126

DOI $10.4171 / \mathrm{GGD} / 178$
Groups, Geometry, and Dynamics

(C) European Mathematical Society

\title{
An upper bound for injectivity radii in convex cores
}

\author{
Brian H. Bowditch
}

\begin{abstract}
Let $N$ be a complete hyperbolic 3-manifold with finitely generated fundamental group, and let $H$ be its convex core. We show that there is an upper bound on the radius of an embedded hyperbolic ball in $H$, which depends only on the topology of $N$. As a consequence, we deduce that limit sets of strongly convergent kleinian groups converge.
\end{abstract}

Mathematics Subject Classification (2010). 57M50.

Keywords. Hyperbolic 3-manifold, convex core, injectivity radius.

\section{Introduction}

Let $N$ be a complete hyperbolic 3-manifold with finitely generated fundamental group. By tameness [Bo], [A], [CalG], it is known that $N$ is homeomorphic to the interior of a compact manifold, $M$. Let $H$ be the convex core of $N$. (This is the minimal submanifold with locally convex boundary whose inclusion into $N$ is a homotopy equivalence.)

The main result will be:

Theorem 0.1. There is some $r \geq 0$, depending only on the topology of $M$, such that any embedded hyperbolic ball in $H$ has radius at most $r$.

The question has been considered by a number of authors. The statement is proven in [Can] for product manifolds, and in [F1], [F2] for books of $I$-bundles and acylindrical manifolds, and in $[\mathrm{E}]$ in the general incompressible case. A proof in the general (tame) case (different from that presented here) was worked out by Kleineidam and Souto, though never written down in detail.

Theorem 0.1 is related to a question of McMullen (posed before the Tameness Theorem was proven in general) in the problem list [Bi]. That question asks whether one can find a radius bound that depends only on the minimal number of generators of $\pi_{1}(M)$. McMullen's question is already interesting, and remains open, when $M$ is closed. Our result applies to general $M$, though we make no attempt to relate the bound to any specific algebraic properties of $\pi_{1}(M)$. 
In fact we prove a variation (Theorem 1.1) which is easily seen to imply Theorem 0.1 . This says that any curve in $H$ either lies in a small compressing disc in $H$, or else lies in an essential curve in $H$ of bounded length. Indeed, by Ahlfors's Finiteness Theorem, the boundary $\partial H$, of $H$ is an intrinsically hyperbolic surface of bounded area, and so it's not hard to see that Theorem 0.1 also implies Theorem 1.1. In other words, they are essentially equivalent.

We also note that there are only finitely many possibilities for the topology of $M$ once its homotopy class is determined. (This is a consequence of the characteristic submanifold construction [J], see [S].) Thus, in Theorem 0.1, one can take $r$ to be a function of the fundamental group.

It was pointed out to me by Al Marden that Theorem 1.1 can be used to prove the convergence of limit sets of a sequence of finitely generated kleinian groups which converge both algebraically and geometrically. This is given here as Theorem 8.1. The argument is fairly straightforward, given a result of McMullen in [M].

We will prove Theorem 1.1 in a series of more general situations. In Sections 3, 4 and 5, we deal with the convex co-compact case. Here, $H$ is respectively a product, compression body, or general compact manifold. In Section 6, we consider the case of a tame manifold without cusps. We will finally deal with the general case in Section 7. Our proof will involve constructing sequences of homotopies and compressions of singular hyperbolic surfaces. Related constructions can be found, for example in $[\mathrm{BrS}]$ and $[\mathrm{BrMNS}]$.

I thank the referees for their helpful comments on this paper.

\section{Convex hulls}

In this Section we make a few relatively simple observations and give a reformulation of the main result, namely Theorem 1.1. We use $\mathbb{H}^{3}$ to denote hyperbolic 3-space, $d$ for its metric, $B(Q, r)$ for the $r$-neighbourhood of $Q \subseteq \mathbb{H}^{3}$.

Let $G$ be a non-cyclic infinite torsion-free group acting properly discontinuously on $\mathbb{H}^{3}$. We write $\widetilde{H}$ for the convex hull of the limit set, and $H=\widetilde{H} / G \subseteq N=$ $\mathbb{H}^{3} / G$ for the convex core of $N$, which we assume to be compact. It follows by Ahlfors's Finiteness Theorem that in the induced path-metric, $\partial H$, is a finite disjoint union of complete hyperbolic surfaces. In the above, we should insert a clause about the "fuchsian" case, where $H$ is a totally geodesic surface with totally geodesic boundary. It is then most natural to view $\partial H$ as the double of $H$. Our statements are easily reinterpreted (though essentially elementary) in that case.

Given $t>0$, let $\mathbb{H}_{\leq t}^{3}(G)=\left\{x \in \mathbb{H}^{3} \mid(\exists g \in G \backslash\{1\})(d(x, g x) \leq t)\right\}$, and set $N_{\leq t}=\mathbb{H}_{\leq t}^{3}(G) / G \subseteq N$. In other words, $N_{\leq t}$ is the set of points of $N$ contained in an essential curve of length at most $t$. If $\eta>0$ is less than the Margulis constant, then each component of $\mathbb{H}_{\leq \eta}^{3}(G)$ has the form $\mathbb{H}_{\leq \eta}^{3}(Z)$ where $Z \leq G$ is a maximal abelian subgroup. The quotient $\mathbb{H}_{\leq \eta}^{3}(Z) / Z \subseteq N$ is a Margulis region. This is either 
a cusp (rank 1 or rank 2) or a Margulis tube. In the last case, we will denote it by $N_{\leq \eta}(\alpha)$, where $\alpha$ is a primitive homotopy class of closed curves in $N$.

We can assume that $\eta$ is also less than the 2-dimensional Margulis constant. If $\xi \in(0, \eta]$ we set $(\partial H)_{\leq \xi}$ to be the " $\xi$-thin" part of $\partial H$. Each component, $A$, of $(\partial H)_{\leq \xi}$ is an annulus with boundary curves $\alpha_{1}, \alpha_{2}$, say, each of length between $\xi$ and $2 \xi$ (provided $\eta$ is sufficiently small). Note that if $\alpha$ is essential in $N$, then $A \subseteq N_{\leq \eta}(\alpha) \subseteq N_{\leq \eta}$.

Suppose that $\alpha$ is trivial in $N$. Then each $\alpha_{i}$ bounds an embedded disc $D_{i} \subseteq H$ of diameter at most $\xi$. We can assume $D_{1}$ and $D_{2}$ to be disjoint, so that $A \cup D_{1} \cup D_{2}$ is a 2-sphere, and so it bounds a ball, $W \subseteq H$, with $W \cap \partial H=A$. We refer to $W$ as a $\xi$-handle in $H$. Note that if $\delta$ is any path from $D_{1}$ to $D_{2}$ in $W$ then $W \subseteq B(\delta, \xi)$.

We write $W(\xi)$ for the union of all $\xi$-handles in $H$ (which we can assume to be disjoint). Note that, up to bounded Hausdorff distance, $W(\xi)$ can be described as the set of points of $H$ contained in some compressing disc of circumference and diameter at most $\xi$. (This ties in with the informal description given in the Introduction.)

We will write $\tau(M)$ for the minimal number of 3-simplices in a triangulation of $M$. This measures the topological compexity of $M$. As a "triangulation" we can allow the image of any $G$-invariant triangulation of $\widetilde{H}$ as a simplicial complex, so that in $H$ we can allow non-embedded simplices, but this makes no essential difference to the argument.

Note that $\tau(M)$ also bounds the complexity of $\partial H$ (for example, as measured as the sum of the genera of its components).

The main result of this paper is:

Theorem 1.1. $(\forall \tau \in \mathbb{N})(\forall \xi>0)(\exists t \geq 0)$ if $M$ can be triangulated with at most $\tau$ 3-simplices (i.e. $\tau(M) \leq \tau$ ), then $H \subseteq N_{\leq t} \cup W(\xi)$, where $W(\xi)$ is the union of all the $\xi$-handles in $H$.

We can refine Theorem 1.1 slightly. If we alter the conclusion to $H \subseteq B\left(N_{\leq t}, r\right) \cup$ $W(\xi)$, then we can choose $t$ to depend only on $\xi$ and the complexity of $\partial H$, though $r$ may also depend on $\tau(M)$. We will see that the proof automatically gives this. Note that $B\left(N_{\leq t}, r\right) \subseteq N_{\leq(t+2 r)}$, and so this implies Theorem 1.1 as stated.

As observed in the introduction, there are only finitely many homeomorphism types for a compact 3-manifold $M$ with given fundamental group. We only really need this when every 2-sphere in $M$ bounds a ball. In the boundary-incompressible case this is a consequence of Corollary 29.3 of [J]. More generally, we can cut along a collection of compressing discs into boundary-incompressible pieces. Their fundamental groups are determined as the maximal one-ended subgroups of the original $\pi_{1}(M)$. Thus, there are only finitely many possibilities for the pieces, and for regluing them to reconstruct $M$. (See [S] for details.) As a consequence of this observation, the complexity, $\tau(M)$, featuring in Theorem 1.1 could, in fact, be taken to be a function of $\pi_{1}(M)$. 


\section{Some definitions and facts}

We begin by recalling or reformulating a few well known facts and constructions used in the proof. We assume here there are no parabolics. We describe how the relevant statements can be modified in Section 7.

Definition. By a singular (hyperbolic) surface in $N$, we mean a 1-lipschitz map, $\phi: \Sigma \rightarrow N$, where $\Sigma$ is a closed surface with a hyperbolic structure.

(We could allow uniformly lipschitz maps, or cone singularities with angles at least $2 \pi$ without essential change.)

We say that a free homotopy class of closed curves in $\Sigma$ is compressing if it is non-trivial in $\Sigma$ but its image in $N$ is trivial. Suppose we fix some $\eta>0$. Then each component of the $\eta$-thin part of $\Sigma$ is either compressing, or maps into the thin part, $N_{\leq \eta}$, of $N$. Also each component of the complement of the $\eta$-thin part of $\Sigma$ has bounded diameter, depending only on $\eta$ and the genus of $\Sigma$.

Definition. By a multisurface we mean a (possibly empty) disjoint union of closed surfaces.

We can generalise the above to singular mutlisurfaces. Note that the inclusion of $\partial H$ into $N$ is a singular multisurface (in fact a non-singular one).

Definition. A multicurve in a multisurface, $\Sigma$, is a disjoint union of homotopically distinct non-trivial closed curves. It is complete if each component of the complement is a three-holed sphere (or "3HS").

In other words, it gives a pants decomposition of each component of $\Sigma$. If $\phi: \Sigma \rightarrow N$ is any homotopy class of maps, we say that $\gamma$ is totally incompressible if each component of $\phi(\gamma)$ is non-trivial in $N$.

Definition. By a realisation of $\gamma$ in $N$ we mean a singular hyperbolic multisurface, $\phi: \Sigma \rightarrow N$, such that $\phi \mid \gamma$ maps each component of $\gamma$ locally isometrically to the corresponding closed geodesic in $N$.

Lemma 2.1. Any totally incompressible multicurve, $\gamma \subseteq \Sigma$, admits a realisation. If $\gamma$ is a complete multicurve, then any two such realisations are connected by a homotopy in $N$ whose image lies in a bounded neighbourhood of the image of either of the realisations.

Proof. These are fairly standard, see for example [Bo].

Definition. We will define the "complexity" of a (multi)surface to be minus the Euler characteristic. 
We also recall Bers's Lemma [Be]:

Lemma 2.2. Given any hyperbolic structure on a closed surface $\Sigma$, there is a complete geodesic multicurve total length is bounded above in terms of the compexity of $\Sigma$.

Clearly this also applies to multisurfaces.

We can assume that such a multicurve contains all closed geodesics in $\Sigma$ of length at most $\eta$.

We also note the following:

Lemma 2.3. Suppose that $\phi: \Sigma \rightarrow N$ is a singular hyperbolic multisurface, and that $\gamma \subseteq \Sigma$ is a totally incompressible complete multicurve of in $\Sigma$. Then we can homotope $\phi$ to a realisation of $\gamma$ in $N$ by a homotopy lying in $B\left(\phi(\Sigma) \cup N_{\leq \eta}, r\right)$, where $r$ depends only on $\eta$ and the complexity of $\Sigma$, and the length of $\gamma$.

Proof. First homotope each component of $\phi(\gamma)$ to the corresponding closed geodesic. This can be done in a bounded neighbourhood of this curve union the corresponding Margulis tubes. We can then homotope so that each curve maps locally injectively. Now extend over each $3 \mathrm{HS}$.

When we apply this, the length of $\gamma$ will be bounded in terms of the complexity of $\Sigma$, so $r$ will depend only on $\eta$ and this complexity.

We recall the notion of an "elementary move" on complete multicurves (corresponding an edge in the pants graph). We say that $\gamma$ and $\delta$ are connected by an elementary move if there are components $\alpha \subseteq \gamma$ and $\beta \subseteq \delta$ such that $\gamma \backslash \alpha=\delta \backslash \beta$ and such that $\alpha \cup \beta$ has a regular neighbourhood that is either a four-holed sphere (4HS) or a one-holed torus (1HT). In these terms, the fact that the pants graph is connnected $[\mathrm{HT}]$ can be expressed as:

Lemma 2.4. If $\gamma$ and $\gamma^{\prime}$ are complete multicurves, then there is a sequence $\gamma=$ $\gamma_{0}, \gamma_{1}, \ldots, \gamma_{n}=\gamma^{\prime}$ of complete multicurves such that each $\gamma_{i+1}$ is obtained from $\gamma_{i}$ by an elementary move.

We can elaborate on the second part of Lemma 2.1:

Lemma 2.5. Suppose that $\gamma$ and $\delta$ are complete multicurves related by an elementary move, and suppose that $\phi, \psi$ are realisations of $\gamma$ and $\delta$ respectively. Then there is a homotopy from $\phi$ to $\psi$ in $N$ lying in a bounded neighbourhood of $\phi(\Sigma) \cup N_{\leq \eta}$.

Proof. We've just got to worry about 1HT's and 4HS's. These are easily dealt with by cutting into simplices with all vertices in the curves.

Finally we note that we can extend a singular surface to a union of long thin handles about short compressing curves. We state this as follows. (It is a generalistion of the construction of $\xi$-handles.) The proof is elementary. 
Lemma 2.6. Suppose that $\phi: \Sigma \rightarrow N$ is a singular hyperbolic multisurface, and that $\alpha \subseteq \Sigma$ is a compressing curve of length less than $\eta$. Let $B$ be a 2-handle (3-ball) attached to $\Sigma$ so that $\partial B$ meets $\Sigma$ in the component of the $\eta$-thin part of $\Sigma$ with core curve $\alpha$. Then we can extend $\phi$ to a map $\phi: \Sigma \cup B \rightarrow N$, so that $\phi(B)$ lies in a bounded neighbourhood $\phi(\Sigma)$ and the intrinsic diameter of each component of $\partial B \backslash \Sigma$ is bounded.

Here the bounds depend only on $\eta$. (We could take $\eta$ itself.)

We will now move on to give proofs of Theorem 1.1 in increasing generality. First we will assume that the manifold $N$ is convex cocompact, that is, the convex core, $H$, is compact.

This will be based on a simple homological principle. We will take $\mathbb{Z}_{2}$-coefficients. If $\partial H$ bounds a singular 3-chain in $N$, then $H$ is precisely the set of points to which the 3-chain maps with degree 1 . We can construct such a 3-chain for example as a continuous map, $\phi: H \rightarrow N$, with $\phi \mid \partial H$ just inclusion of $\partial H$ in $N$.

\section{The convex cocompact product case}

We consider first the case where $H \cong \Sigma \times[-1,1]$, where $\Sigma$ is a closed surface. We write $\Sigma^{ \pm}=\Sigma \times\{ \pm 1\}$, so that $\partial H=\Sigma^{-} \cup \Sigma^{+}$. This case follows from well known constructions related to interpolating pleated surfaces. It was originally described in [Can]. We describe one such construction which we adapt later. (In this case, we can measure the complexity of $M$ in terms of genus $(\Sigma)$.)

Let $\gamma^{ \pm}$be a complete multicurve in $\Sigma^{ \pm}$of length bounded in terms of genus $(\Sigma)$ (Lemma 2.2). Here all curves are incompressible. Let $\phi^{ \pm}: \Sigma \rightarrow N$ be a realisation of $\gamma^{ \pm}$. By Lemmas 2.1 and 2.3, we can find a homotopy from $\Sigma^{ \pm} \hookrightarrow N$ to $\phi^{ \pm}: \Sigma \rightarrow N$ which lies in a bounded neighbourhood of $\Sigma^{ \pm} \cup N_{\leq \eta}$. Now let $\gamma^{-}=\gamma_{0}, \gamma_{1}, \ldots, \gamma_{n}=$ $\gamma^{+}$be a sequence of complete multicurves as given by Lemma 2.4. Let $\phi_{i}: \Sigma \rightarrow$ $N$ be a realisation of $\gamma_{i}$. By Lemma 2.5, there is a homotopy from $\phi_{i}$ to $\phi_{i+1}$ lying in a bounded neighbourhood of $\phi_{i}(\Sigma) \cup N_{\leq \eta}$. (Note that we don't need a bound on the length of the geodesic realisation of $\gamma_{i}$ for this.) Assembling all the homotopies gives us a homotopy from $\Sigma^{-}$to $\Sigma^{+}$lying in a bounded neighbourhood of $\partial H \cup \bigcup_{i} \phi_{i}(\Sigma) \subseteq N$. We can view this as a map $\phi: H \rightarrow N$, with $\phi \mid \partial H$ just inclusion $\partial H \hookrightarrow N$. Also, for each $i, \phi_{i}(\Sigma) \subseteq N_{\leq t_{0}}$ where $t_{0}$ depends only on genus $(\Sigma)$. (Since, for each $i$, each point of $\Sigma$ in the intrinsic hyperbolic metric lies in a curve of bounded length.) Thus, $H \subseteq \phi(H) \subseteq N_{\leq t}$, where $t$ depends only on genus $(\Sigma)$ as claimed. 


\section{The convex cocompact compression body case}

We next move on the case where $H$ is a compact compression body, which we can assume not to be a product in the above sense. This has outer and inner boundaries $\partial^{+} H$ and $\partial^{-} H$ respectively. Thus, $\partial^{+} H$ is a compressible surface, and $\partial^{-} H$ is a (possibly empty) incompressible multisurface. (Here, the complexity of $M$ can be measured in terms of genus $\left(\partial^{+} H\right)$.)

Our aim again will be to construct a map $\phi: H \rightarrow N$, with $\phi \mid \partial H$ just inclusion, $\partial H \hookrightarrow N$, using a series of singular hyperbolic multisurfaces, $\phi_{i}: \Sigma_{i} \rightarrow N$, for $i=0,1, \ldots, n$. This time, we will set $\phi_{0}$ and $\phi_{n}$ to be respectively the inclusions of $\partial^{+} H$ and $\partial^{-} H$ into $N$. Each $\phi_{i+1}$ will be obtained from $\phi_{i}$ either by a compression, as defined below, or by a homotopy. For topological reasons, there can only be boundedly many compressions (in terms of genus $\left(\partial^{+} H\right)$ ). Each of these compressions and homotopies will lie in a bounded neighbourhood of $\phi_{i}\left(\Sigma_{i}\right) \cup N_{\leq \eta}$. (Here, the bound depends on genus $\left(\partial^{+} H\right)$ and $\eta$. $)$ The strategy will be to perform compressions along "short" compressing curves whenever we have the opportunity. In this way, by going back a bounded number of times, we see that each $\phi_{i}$ is obtained from some $\phi_{j}$ where $j \leq i$ by a series of consecutive such compressions and where either $j=0$, or where $\phi_{j-1}$ has no short compressing discs. In the former case, we note that $\phi_{0}\left(\Sigma_{0}\right)=\partial^{+} H \subseteq N_{\leq t_{0}} \cup W(\xi)$, where $t$ depends only on genus $\left(\partial^{+} H\right)$ and $\xi$. In the latter case, we note that the intrinsically $\eta$-thin part of $\Sigma_{j-1}$ maps into $N_{\leq \eta}$ (since there are no short compressions) and so $\phi_{j-1}\left(\Sigma_{j-1}\right) \subseteq N_{\leq t_{0}}$, with $t_{0}$ depending only on genus $\left(\partial^{+} H\right)$. Assembling $\phi$ out of these maps, we get $H \subseteq \phi(H) \subseteq N_{\leq t} \cup W(\xi)$, where $t$ depends only on genus $\left(\partial^{+} H\right)$ and $\xi$ as required.

We begin by describing the notion of compression in topological terms. By a compression of a multisurface, $\Sigma$, we mean a disjoint union of compression bodies, $C$, such that $\Sigma$ is the union of their outer boundaries. We assume that there are no sphere or torus inner boundary components. We also assume that $C$ is not a product, and so the inner boundary has strictly lower complexity than the outer boundary. Note that we can connect a sequence of such compressions into a single compression.

Suppose $\phi: \Sigma \rightarrow N$ is any map, and $\beta \subseteq \Sigma$ is a totally compressing multicurve (i.e. each component is compressing). We can construct a compression as follows. First glue a disc to each component of $\beta$ and then thicken up the resulting 2-complex into a compression body. This admits a natural map into $N$ up to homotopy. We now cap off each spherical inner boundary component with a 3-ball. We also cap off each toroidal inner boundary component with a solid torus, in such a way that the map of the torus into $N$ extends over the solid torus. (This is always possible since there are no rank-2 free abelian subgroups of $\pi_{1}(N)$.) This gives another compression body, $C$, with inner boundary, $\Sigma^{\prime}$, say. We can extend $\phi$ over $C$ and so in particular get a map of $\Sigma^{\prime}$ into $N$.

Definition. We say that such a manifold, $C$, together with the map, $\phi$, is a compression $\Sigma$, in $N$, which compresses the multicurve, $\beta$. 
Here is another way of describing a compression in $N$, which is topologically equivalent, and which is how we will carry it out geometrically.

Suppose that $\gamma \subseteq \Sigma$ is a complete multicurve. Let $\beta \subseteq \gamma$ be the union of all compressing curves in $\gamma$. Let $\mathcal{P}$ be the set of components of $\Sigma \backslash \gamma$. We write $\mathcal{P}=\mathcal{P}_{0} \sqcup \mathcal{P}_{1} \sqcup \mathcal{P}_{3}$ where $\mathcal{P}_{i}$ has exactly $i$ boundary components in $\beta$. (We count as 2 any pair of boundary components that get identified to the same curve of $\gamma$.) We first construct a multisurface $\Sigma^{1}$ by cutting $\Sigma$ along $\beta$ and gluing in a disc to each boundary component arising. Thus, each $P \in \mathcal{P}_{3}$ gives rise to a 2 -sphere component of $\Sigma^{1}$. Each element of $\mathcal{P}_{1}$ turns into an annulus in $\Sigma^{1}$. There may be torus components of $\Sigma^{1}$ each of which consists of a closed circuit of such annuli. The remaining "hyperbolic" components of $\Sigma^{1}$ all have genus at least 2. Now the union of all closed annuli arising from $\mathcal{P}_{1}$ will give us, in addition to tori, a number (possibly 0 ) of closed annuli in the hyperbolic components. For each such annulus, $A$, we perform another surgery by cutting along the boundary components and regluing in pairs, so as to give a torus (arising from $A$ ) and a disjoint surface homeomorphic to the original. We thus arrive at another multisurface, $\Sigma^{2}$. We finally throw away all the sphere and torus components of $\Sigma^{2}$ to give us a multisurface, $\Sigma^{\prime}$. Each of the above multisurfaces comes with a natural homotopy class of map to $N$. Also, the multicurve $\gamma$ in $\Sigma$ gives rise to a complete multicurve $\gamma^{\prime}$ in $\Sigma^{\prime}$. (In constructing $\gamma^{\prime}$, we throw away the components of $\beta$, and identify certain pairs of curves in $\gamma \backslash \beta$.) It's not hard to see that $\Sigma \sqcup \Sigma^{2}$ bounds a compression body in a natural way. To get to $\Sigma^{\prime}$ it remains to cap off the 2-spheres with 3-balls, and tori with solid tori. (Note that if $F$ is a torus component of $\Sigma^{2}$, then the image of $\pi_{1}(F)$ in $\pi_{1}(N)$ is non-trivial by construction, and hence contained in a unique maximal cyclic subgroup. There is thus a canonical way to glue in the solid torus so as to kill the kernel.) By construction, we get a compression of $\Sigma$ to $\Sigma^{\prime}$ in $N$, in the same sense as described earlier. Note also by construction, each curve of $\gamma^{\prime}$ is non-compressing on $\Sigma^{\prime}$.

Before applying the above we need a couple of geometrical observations. The proofs are straightforward.

Lemma 4.1. (1) Any map $\phi: F \rightarrow N$ of a 2-sphere, $F$, into $N$ extends to a map $\phi: B \rightarrow N$ of the 3-ball, with the diameter of $\phi(B)$ bounded above by the intrinsic diameter of $F$ in the induced metric.

(2) Any map $\phi: F \rightarrow N$ of a torus $F$, into $N$ extends to a map of a solid torus, $D$, into $N$ such that $\phi(D)$ lies in a bounded neighbourhood of $\phi(F) \cup N_{\leq \eta}$, where the bound depends on the intrinsic diameter of $F$ and $\eta$.

Will be using two geometrically distinct forms of compression, which we will term "short" and "long".

Short compression. Suppose that $\phi: \Sigma \rightarrow N$ is a singular multisurface (that is 1-lipschitz with respect to some hyperbolic structure on $\Sigma$ ). Suppose that $\gamma \subseteq \Sigma$ is an intrinsically geodesic complete multicurve of bounded length (Lemma 2.2). It is 
assumed to contain all closed geodesics of length at most $\eta$. Let $\beta \subseteq \gamma$ be the union of all compressing curves in $\gamma$. We say that $\Sigma$ "admits a short compression" if there is some such $\gamma$ for which $\beta$ is non-empty. In this case, let $\gamma^{\prime} \subseteq \Sigma^{\prime}$ be as described above, and let $\phi^{\prime}: \Sigma^{\prime} \rightarrow N$ be a realisation of $\gamma^{\prime}$. (Recall that no component of $\gamma^{\prime}$ is compressing.) We can now interpolate between $\phi$ and $\phi^{\prime}$ by a compression whose image lies in a bounded neighbourhood of $\phi(\Sigma) \cup N_{\leq \eta}$ as follows. If $\alpha$ is a component of $\beta$, then we span $\phi(\alpha)$ by a disc of bounded diameter if its length is greater than $\eta$, or we attach a handle as given by Lemma 2.6 , if its length is greater than $\eta$. If $\delta$ is a component of $\gamma \backslash \beta$ giving rise to a component of $\gamma^{\prime}$, then homotope $\phi(\delta)$ to its geodesic realisation in $N$ by a homotopy lying in a bounded neighbourhood of $N_{\leq \eta}$. To extend to a compression it remains to cap off a collection of 2 -spheres and tori, all of which have bounded intrinsic diameter. This can be achieved using Lemma 4.1.

Long compression. Suppose that $\phi: \Sigma \rightarrow N$ is a realisation of a complete multicurve $\gamma \subseteq \Sigma$. Suppose that $\delta$ is another multicurve obtained from $\gamma$ by an elementary move, as defined in Section 2. In other words, we have components, $\alpha$ of $\gamma$ and $\beta$ of $\delta$, lying in the same component, $F$, of $\Sigma \backslash(\gamma \backslash \alpha)=\Sigma \backslash(\delta \backslash \beta)$, and such that $\alpha$ and $\beta$ have minimal intersection in $F$. Suppose that $\beta$ is compressible. Then $F$ must be a 4HS. (If it were a $1 \mathrm{HT}, \partial F \subseteq \gamma$ would also be compressing, contradicting the existence of a realisation of $\gamma$ in $N$.)

We now perform a compression of $\beta$. This involves removing $F$ and reconnecting the boundary curves in pairs.

More precisely, suppose that $\beta$ cuts $F$ into 3HS's, $E_{1}$ and $E_{2}$, with $\partial E_{i}=$ $\beta \cup \epsilon_{i} \cup \epsilon_{i}^{\prime}$, and that $\alpha$ cuts $F$ into the 3HS's, $E$, $E^{\prime}$, with $\partial E=\alpha \cup \epsilon_{1} \cup \epsilon_{2}$ and $\partial E^{\prime}=\alpha \cup \epsilon_{1}^{\prime} \cup \epsilon_{2}^{\prime}$. Note that $\epsilon_{i}$ and $\epsilon_{i}^{\prime}$ are homotopic, after compression of $\beta$, and so have the same geodesic realisations, $\phi\left(\epsilon_{i}\right)$ and $\phi\left(\epsilon_{i}^{\prime}\right)$, in $N$. We can therefore throw away $F$ and glue together each $\epsilon_{i}$ and $\epsilon_{i}^{\prime}$ to give a new multisurface, $\Sigma^{\prime}$, and a realisation, $\phi^{\prime}: \Sigma^{\prime} \rightarrow N$, of a complete multicurve $\gamma^{\prime}$ in $\Sigma^{\prime}$. (We need to consider the possibility that $\epsilon_{i}=\epsilon_{i}^{\prime}$, in which case we just discard this curve. If this holds for both $i=1,2$, then we end up discarding the entire genus 2 component of $\Sigma$ containing $\alpha$.) Note that $\phi^{\prime}\left(\Sigma^{\prime}\right) \subseteq \phi(\Sigma)$, and that $\phi^{\prime}\left(\Sigma^{\prime}\right)$ is obtained from $\phi(\Sigma)$ by a compression of $\beta$. Topologically, this entails thickening up $F$ on the inside, and gluing in a 2-handle so as to give a genus 2 handlebody, with $F$ in its boundary. Now, $F=E \cup E^{\prime}$, and $\phi(\partial E)=\phi\left(\partial E^{\prime}\right)=\phi(\alpha) \cup \phi\left(\epsilon_{i}\right) \cup \phi\left(\epsilon_{i}^{\prime}\right)$. To map in the handlebody, we can use a homotopy between $\phi(E)$ and $\phi\left(E^{\prime}\right)$ fixing $\phi(\partial E)$. As in Lemma 2.5, such a homotopy can be carried out within a bounded neighbourhood of $\phi(F)$.

Note that this construction does not require any bound on the lengths of the realisation of $\gamma$. We refer to the procedure as a "long compression".

Construction of singular multisurfaces. We now set about constructing the sequence of singular multisurfaces described at the beginning of this section. 
We start with $\Sigma_{0}=\partial^{+} H$ and let $\phi_{0}: \Sigma_{0} \rightarrow N$ be inclusion. We construct a map $\phi_{1}: \Sigma_{1} \rightarrow N$ as follows. If $\phi_{0}$ admits a short compression then we carry it out to obtain a map $\phi_{1}: \Sigma_{1} \rightarrow N$, which realises some multicurve, $\gamma_{1} \subseteq \Sigma_{1}$. If $\phi_{0}$ admits no such compression, then we choose a multicurve, $\gamma_{0}$, in $\Sigma_{0}$ of bounded length. In this case, we set $\Sigma_{1}=\Sigma_{0}$ and $\gamma_{1}=\gamma_{0}$, and we let $\phi_{1}: \Sigma_{1} \rightarrow N$ be a singular surface realising $\gamma_{1}$ (cf. the product case, Section 3).

We now proceed inductively. Suppose we have $\phi_{p}: \Sigma_{p} \rightarrow N$, realising $\gamma_{p}$ with $p \geq 1$. If $\phi_{p}\left(\Sigma_{p}\right)$ admits a short compression, then we carry it out to give a new map $\phi_{p+1}: \Sigma_{p+1} \rightarrow N$ realising a complete multicurve $\gamma_{p+1}$. (Note that the multicurve we use for the short compression depends only on the metric on $\Sigma_{p}$, and need bear no relation to $\gamma_{p}$.)

Suppose that $\phi_{p}\left(\Sigma_{p}\right)$ does not admit a short compression, but that $\phi_{p}\left(\Sigma_{p}\right)$ is not incompressible. Let $\beta \subseteq \Sigma_{p}$ be any compressing curve. Let $S \subseteq \Sigma_{p}$ be the component containing $\beta$. We extend $\beta$ arbitrarily to a complete multicurve, and connect this to $\gamma_{p} \cap S$ by a path $\gamma_{p} \cap S=\delta_{0}, \delta_{1}, \ldots, \delta_{q} \supseteq \beta$ in the pants graph (Lemma 2.4). We extend each $\delta_{j}$ to a complete muticurve in $\Sigma_{p}$, also denoted $\delta_{j}$ by setting $\delta_{j} \backslash S=\gamma_{p} \backslash S$ for all $j \in 0,1, \ldots, q$. (In other words, we leave the other components of $\Sigma_{p}$ alone.) We can assume that $\delta_{j}$ is totally incompressible for all $j<q$ (otherwise we just stop there, and reset $q=j$ ).

We now construct maps $\phi_{p+i}: \Sigma_{p+i} \rightarrow N$, with $\Sigma_{p+i}=\Sigma_{p}$ realising $\gamma_{p+i}=\delta_{i}$ until we either arrive at a surface admitting a short compression, or arrive at $\gamma_{p+q-1}=$ $\delta_{q-1}$. In the former case, we carry out the short compression, and start again with a new multisurface of lower complexity playing the role of $\Sigma_{p}$ above. If there if there is never any short compression in this sequence, then we finally carry out a long compression to $\phi_{p+q-1}\left(\Sigma_{p+q-1}\right)$, compressing the curve $\beta \subseteq \gamma_{p+q}$ and start again from the new multisurface.

After a bounded number of compressions, we eventually arrive at some map $\phi_{m}: \Sigma_{m} \rightarrow N$, with $\phi_{m}\left(\Sigma_{m}\right)$ incompressible in $N$. (Possibly $\Sigma_{m}=\emptyset$.)

Now $\phi_{m}\left(\Sigma_{m}\right)$ is homotopic to $\partial^{-} H$. We now proceed as in the product case (for each component of $\Sigma_{m}$ ) so as to construct maps $\phi_{m+i}: \Sigma_{m+i} \rightarrow N$, with $\Sigma_{m+i}=\Sigma_{m}$ for all $i$, terminating with a map $\phi_{n}: \Sigma_{n} \rightarrow N$, which is just the inclusion of $\partial^{-} H$ into $N$.

Assembling all the $\phi_{i}$, we get a map $\phi: H \rightarrow N$, with $\phi \mid \partial H$ just inclusion, as discussed at the beginning of this section.

This proves Theorem 1.1, in the case where $H$ is a compact compression body.

\section{General cocompact case}

We explain how to reduce the general cocompact case to the previous cases dealt with. A similar principle is used in [E].

Let $N$ be a complete hyperbolic manifold with compact convex core, $H$. The inclusion $H \hookrightarrow N \hookrightarrow M$ is homotopic to a homeomorphism. Indeed, $M \backslash H \cong$ $\partial M \times[0, \infty)$. 
Let $S$ be a boundary component of $H \cong M$. Let $N_{S}$ be the cover of $N$ corresponding to $S \hookrightarrow N$. We lift $S$ to an injective map $S \rightarrow N_{S}$. This is a boundary component of the convex core, $H_{S} \subseteq N_{S}$.

Now $N_{S}$ is also convex cocompact. This is a standard argument due to Thurston. (By Ahlfors's Finiteness Theorem, $\partial H_{S}$ is compact. Since $H$ is compact, every point must lie a bounded distance from its boundary, and so the same is true of $H_{S}$. Thus, $H_{S}$ is compact.) Since $S \hookrightarrow H_{S}$ is surjective on fundamental groups, $H_{S}$ is a compression body.

Suppose we are given a triangulation of $S$. Then we can construct a piecewise straight map, $\psi_{S}$, of $S$ into $N_{S}$, homotopic to inclusion. By "piecewise straight" we mean that each simplex gets mapped to a totally geodesic simplex in $N$. We can do this by mapping in vertices, edges and triangles in turn. In fact, by choosing this so as to minimise the total length of the 1-skeleton, we will have $\psi_{S}(S) \subseteq H_{S}$. (Otherwise there will be a vertex $x$ in $S$, whose link gets mapped strictly into a hemisphere in the unit tangent space in $N_{S}$. This would allow us to shorten the 1-skeleton by pushing the vertex in the direction of the centre of the hemisphere.) We refer to piecewise straight maps of this sort as "balanced".

Let $W_{S}(\xi)$ be the union of all $\xi$-handles in $H_{S}$, and $\left(N_{S}\right)_{\leq t}$ be the set of points contained in an essential loop of length at most $t$ in $N_{S}$. By the earlier cases (Sections 3 and 4), we have $H_{S} \subseteq\left(N_{S}\right)_{\leq t_{S}} \cup W_{S}(\xi)$, where $t_{S}$ depends only on the genus of $S$. In particular, we get a $\mathbb{Z}_{2}$-homology chain bounded by $\psi_{S}(S) \cup S$ in $N_{S}$ and whose image lies in $\left(N_{S}\right)_{\leq t_{S}} \cup W_{S}(\xi)$. Mapping down to $N$, we get a map $\psi: S \rightarrow N$ with $\psi(S) \cup S$ bounding a homology chain lying in $N_{\leq t_{S}} \cup W(\xi)$. Let $P_{S}$ be the set of point to which this maps with degree 1 .

We now take a triangulation of $M \cong H$ with at most $\tau 3$-simplices. This induces a triangulation on $\partial H$. We perform the above construction for each component of $\partial H$, to give a piecewise straight map $\psi: \partial H \rightarrow N$ (mapping in the remaining vertices, edges, triangles and simplices in turn). We extend this to a piecewise straight map $\psi: H \rightarrow N$. Let $Q \subseteq N$ be the set of points to which it maps with degree 1 (in $\mathbb{Z}_{2}$ ). The volume of $Q$ is bounded above by $\tau$ times the volume of the regular ideal 3 -simplex. This places a bound, say $r$, depending only on $\tau$, on the radius of the largest hyperbolic ball we can embed in $Q$.

Now the homology 3-chains with boundaries $\psi(S) \cup S$ combine to give us a 3chain with boundary $\phi(\partial H) \cup \partial H$. Combining this with $\psi(H)$ we get a 3-chain with boundary $\partial H$. This maps with degree 1 to $H$, and so we see that $H=Q \cup \cup_{S} P_{S}$ as $S$ ranges over the boundary components. It follows that $H \subseteq N_{\leq t} \cup W(\xi)$, where $t=2 r+\max _{S} t_{S}$. This proves Theorem 1.1 in the general convex cocompact case. (Note that this includes the cocompact case, where $M=H$ is a closed manifold, and $\partial H=\emptyset$.) 


\section{Geometrically tame without parabolics}

Let $N$ be a complete hyperbolic 3-manifold with without parabolics. By tameness [Bo], [A], [CalG], $N$ is homeomorphic to the interior of a compact manifold, $M$. We can embed $M$ into $N$ so that $N \backslash$ int $M \cong \partial M \times[0, \infty)$. Let $S$ be a component of $\partial M$, so that $E_{S} \equiv S \times[0, \infty)$ is an end of $N$. This end is either geometrically finite, in which case we can assume that $S$ is a boundary component of $\partial H$, or else simply degenerate, in which case, we can assume that $E_{S} \subseteq H$.

Our aim in this case will be to construct a homology 3-chain whose boundary consists of $\partial H$ together with compact subsets lying arbitrarily far out each of the degenerate ends. This chain will map with degree 1 to an arbitrarily large compact subset of $H$.

Suppose that $E$ is simply degenerate. In this case, there is a sequence, $\omega^{n}: S \rightarrow$ $E_{S}$, of singular hyperbolic surfaces, each homotopic in $E_{S}$ to the inclusion of $S=\partial E_{S}$, and tending out the end. Indeed we can take $\omega^{n}$ to be the realisation of some complete multicurve, $\gamma^{n}$, in $S$. Suppose $\beta$ is any curve $S$ such that $\omega^{n}(\beta)$ is compressing in $N$. There is a compressing disc in $N$ whose diameter is bounded above by length $\left(\omega^{n}(\beta)\right)$. This disc must meet $H$. It follows that the length of the shortest compressing curve in $\omega^{n}(S)$ (if there is any) must tend to $\infty$ as $n \rightarrow \infty$. In particular, we can assume that none of the surfaces $\omega^{n}(S)$ admits any short compression (in the sense described in Section 4).

Now suppose that $x \in H$ is any point in the convex core. For each simply degenerate end, $E_{S}$, we choose a singular $\omega_{S}: S \rightarrow E_{S}$ with $x$ lying in the same component of $E_{S} \backslash \omega_{S}(S)$ as $S=\partial E_{S}$. (Set $\omega_{S}=\omega^{n}$ for large enough $n$.) This realises a complete multicurve $\gamma_{S}$. Note in particular, that $\omega_{S}(S)$ homologically separates $x$ from the end. If $E_{S}$ is geometrically finite, we set $\omega_{S}$ to be the inclusion $S \hookrightarrow N$. Combining these gives us a map $\omega: \partial M \rightarrow N$. We aim to extend this to a homology 3-chain with boundary $\omega(\partial M)$. This maps with degree 1 to $x$. For this, we use a piecewise straight map, $\psi: M \rightarrow N$, with $\psi \mid \partial M$ balanced, constructed as in Section 5 . We need to find a chain with boundary $\psi(S) \cup \omega(S)$ for each component, $S$, of $\partial M$. We follow the procedure of previous sections.

Suppose, for example, that $E_{S}$ is a simply degenerate end. As in Section 5, we lift to $N_{S}$, which is the interior of a compact product manifold or compression body, $M_{S}$, with outer boundary $\partial^{+} M_{S} \cong S$. The outer end is simply degenerate and isometric to $E_{S}$. We can lift $\omega_{S}: S \rightarrow E_{S}$ to a map to $N_{S}$, also realising the complete multicurve $\gamma_{S}$. Since $\omega_{S}(S)$ has no short compression, we can immediately set about searching for a long compression similarly as in Section 4, using Lemma 2.4, starting with $\gamma_{S}$. Moreover, if $\omega_{S}(S)$ is sufficiently far out the end, then $\omega_{S}(S)$ lies in the convex core, $H_{S}$, of $N_{S}$. The lift, $\psi_{S}(S)$, of $\psi(S)$ to $N_{S}$ also lies in $H_{S}$ (since it is a balanced piecewise straight map). We can therefore find a homology chain with boundary $\psi_{S}(S) \cup \omega_{S}(S)$ in $N_{S}$. As in Section 4, this lies in $\left(N_{S}\right)_{\leq t_{S}}$ where $t_{S}$ depends only on genus $(S)$. If $E_{S}$ is geometrically finite, we similarly obtain a homology chain lying in $\left(N_{S}\right)_{\leq t_{S}} \cup W(\xi)$. 
Mapping down to $M$, we end up constructing a $\mathbb{Z}_{2}$-homology 3-chain, with boundary $\omega(\partial M)$, and supported on $N_{\leq t} \cup W(\xi)$, where $t$ depends only on $\tau$ and $\xi$. This chain also maps to $x$ with degree 1 , and so $x \in N_{\leq t} \cup W(\xi)$. Since $x \in H$ was arbitrary, we deduce that $H \subseteq N_{\leq t} \cup W(\xi)$, as required.

\section{The general case}

To deal with the general case, where we allow for cusps, a few modifications are necessary. We need to adapt the definition of "singular surface" to allow for nodal surfaces. We need to consider $\mathbb{Z} \oplus \mathbb{Z}$-cusps. Also, the $\mathbb{Z}$-cusps may cut a topological end of $M$ into pieces, some of which may be geometrically finite while others simply degenerate.

Let $N$ be a complete hyperbolic 3-manifold with $\pi_{1}(N)$ finitely generated. Again by tameness, we can embed $M$ into $N$, so that $N \backslash$ int $M \cong \partial M \times[0, \infty)$. The $\mathbb{Z} \oplus \mathbb{Z}$ cusps are in bijective correspondence to the toroidal components of $\partial M$, and each can be assumed to be the boundary of the corresponding $\eta$-Margulis cusp. Let $\partial_{0} M$ be the union of all the components of genus at least 2. Tameness also tells us that there is a multicurve, $\pi \subseteq \partial_{0} M$, with the $\mathbb{Z}$-cusps in bijective correspondence with the components of $\pi$.

More precisely, if $\alpha \subseteq \partial_{0} M$ is a component of $\pi$, then there is an $\eta$-Margulis cusp, $N_{\leq \eta}(\alpha)$, homotopic to $\alpha$ in $N$. We can assume that $\partial M$ meets $N_{\leq \eta}(\alpha)$ in an annulus $A(\alpha)$ with core curve $\alpha$.

We write $N_{\leq \eta}^{\text {nc }} \subseteq N$ for the complement of the interiors of all the $\eta$-Margulis cusps.

Definition. We refer to $N_{\leq \eta}^{\text {nc }}$ as the non-cuspidal part of $N$.

We write $\mathscr{F}$ for the set of components of $\partial_{0} M \backslash \bigcup_{\alpha \subseteq \pi}$ int $A(\alpha)$ (which we can identify with the "non-cuspidal" part of $\partial N_{\leq \eta}^{\mathrm{nc}}$ ). Each component of $N_{\leq \eta}^{\mathrm{nc}} \backslash \operatorname{int} M$ has the form $E_{F} \cong F \times[0, \infty)$, where $F$ is identified with $F \times\{0\}-$ the relative boundary of $E_{F}$ in $N_{\leq \eta}^{\text {nc }}$. These are the non-cuspidal "geometric" ends of $N$. Each is either geometrically finite or simply degenerate, and we partition $\mathcal{F}_{F}=\mathscr{F}_{F} \sqcup \mathscr{F}_{D}$ accordingly. If $F \in \mathscr{F}_{D}$ then $E_{F} \subseteq H$.

By Ahlfors's Finiteness Theorem, $\partial H$ is an intrinsically hyperbolic multisurface of finite area. Moreover, each cusp of $\partial H$ maps into a $\mathbb{Z}$-cusp of $N$. Choosing $\eta$ sufficiently small in relation to the topological type of $\partial H$ (which is bounded in terms of the complexity of $M$ ) we can assume that $\partial H$ meets the $\mathbb{Z}$-cusp in a horodisc of $\partial H$ which is totally geodesic in $N$. (It is possible that $\partial H$ might meet other cusps in a compact subset, but that does not matter in what follows.)

We will need to generalise the notion of a singular hyperbolic surface in $N$, to allow for curves to be sent off to infinity in parabolic cusps. 
Let $\Sigma$ be a multisurface. By a nodal structure in $\Sigma$, we mean a (possibly empty) multicurve, $\zeta$, together with a complete finite-area hyperbolic structure on $\Sigma \backslash Z$, where $Z$ is a closed regular neighbourhood of $\zeta$. We also note that Bers's Lemma also applies to finite area surfaces, and therefore also to nodal structures. In other words, we can extend $\zeta$ to a complete multicurve in $\Sigma$ so that the length of each other component is bounded above in terms of genus $(\Sigma)$.

To define a singular nodal surface, we embed $N$ as a submanifold of a manifold $N \cup \partial^{\mathrm{c}} N$ with boundary $\partial^{\mathrm{c}} N$, where each component of $\partial^{\mathrm{c}} N$ corresponds to a cusp. Formally, we can view $\partial^{\mathrm{c}} N$ as a disjoint copy of $\partial N_{\leq \eta}^{\mathrm{nc}}$, and topologise $N \cup \partial^{\mathrm{c}} N$ in such a way that $N \cup \partial^{\mathrm{c}} N \backslash$ int $N_{\leq \eta}^{\mathrm{nc}}$ is a product, $\partial N_{\leq \eta}^{\mathrm{nc}} \times[0, \infty]$ with $\partial N_{\leq \eta}^{\mathrm{nc}} \equiv \partial N_{\leq \eta}^{\mathrm{nc}} \times\{0\}$ and with $\partial^{\mathrm{c}} N \equiv \partial N_{\leq \eta}^{\mathrm{nc}} \times\{\infty\}$, and such that each $\{x\} \times[0, \infty)$ is a geodesic ray. Note that the ends of $N \cup \partial^{\mathrm{c}} N$ are in bijective correspondence with the ends of $N_{\leq \eta}^{\mathrm{nc}}$

Definition. A (singular) nodal surface is a map $\phi: \Sigma \rightarrow N \cup \partial^{\mathrm{c}} N$ such that $Z=$ $\phi^{-1}\left(\partial^{\mathrm{c}} N\right)$ is a regular neighbourhood of a multicurve in $\Sigma$, and such that $\phi \mid(\Sigma \backslash Z)$ is 1-lipschitz with respect to a nodal structure corresponding to the multicurve.

Only $\phi \mid(\Sigma \backslash Z)$ is relevant to geometric arguments. We need $\phi$ defined on $Z$ to enable us to define homotopy and homology classes.

The observations of Section 2 go through with little change, with "singular hyperbolic surface" replaced by "nodal surface".

To explain how the proof is modified, we work backwards through the paper.

In Section 6, we need to take account to the possibility that an end of $N$ may have both geometrically finite and simply degenerate parts. Suppose that $F \in \widetilde{F}_{D}$, i.e. $E_{F}$ is simply degenerate. In this case we have a sequence, $\left(\omega^{n}\right)_{n}$ of 1-lipschitz maps into $M$ where the domain of each is a finite area surface homeomorphic to int $F$. The image of $\omega^{n}$ meets $N_{\leq \eta}^{\text {nc }}$ in a compact subset of $E_{F}$. This homologically separates $F$ from the end of $E_{F}$. Moreover, these compact subsets tend out the end as $n \rightarrow \infty$. On the other hand, if $F \in \mathcal{F}_{F}$, we can set each $\omega^{n}$ to be equal to the inclusion of the corresponding component of $\partial H$ into $N$. Piecing together these maps with annuli in $\partial^{\mathrm{c}} N$, we get a nodal surface $\omega^{n}: \partial_{0} M \rightarrow N \cup \partial^{\mathrm{c}} N$, based on the multicurve $\pi$ determined by the $\mathbb{Z}$-cusps. We extend this topologically to a map $\omega^{n}: \partial M \rightarrow N \cup \partial^{\mathrm{c}} N$, taking each (toroidal) component of $\partial M \backslash \partial_{0} M$ to a component of $\partial^{\mathrm{c}} N$.

Let $Q^{n} \subseteq N$ be the set of points $\mathbb{Z}_{2}$-homologically separated from infinity by $\omega^{n}\left(\partial_{0} M\right)$, in other words, the set of points of $N$ to which $\omega^{n}: \partial M \rightarrow N \cup \partial^{\mathrm{c}} N$ maps with degree 1 . By construction, $N_{\leq \eta}^{\text {nc }} \cap H \subseteq \bigcup_{n} Q^{n}$.

Let $S$ be a component of $\partial_{0} M$. Let $N_{S}$ be the corresponding cover. We define $\partial^{\mathrm{c}} N_{S}$ similarly as for $\partial^{\mathrm{c}} N$. There is a natural map $N_{S} \cup \partial^{\mathrm{c}} N_{S} \rightarrow N \cup \partial^{\mathrm{c}} N$ which bijective on each component of $\partial^{\mathrm{c}} N_{S}$. As before, by tameness, $N_{S}$ is the interior of a compact manifold, which must be a compression body (or product) with outer boundary corresponding to $S$. This time there might be toroidal inner boundary components corresponding to $\mathbb{Z} \oplus \mathbb{Z}$-cusps. Let $H_{S}$ be the convex core of $N_{S}$. If 
we assume the compression body case (which will be explained below) then $H_{S} \subseteq$ $\left(N_{S}\right)_{\leq t_{S}} \cup W_{S}(\xi)$, where $t_{S}$ depends only on the genus of $S$.

As before, we can construct a balanced piecewise straight map, $\psi_{S}: S \rightarrow N_{S} \cup$ $\partial^{\mathrm{c}} N_{S}$, which in this case may be a nodal surface. We will have $\psi_{S}(S) \cup N \subseteq H_{S}$. Let $\omega_{S}^{n}: S \rightarrow N_{S} \cup \partial^{\mathrm{c}} N_{S}$ be the lift of $\omega^{n} \mid S$. We can assume that $\omega_{S}^{n}(S) \cap N_{S} \subseteq H_{S} \cup$ $\left(N_{S}\right)_{\leq \eta}$. Now $\omega_{S}^{n}$ is homotopic to $\psi_{S}$. Let $P_{S}^{n} \subseteq N_{S}$ be the set of points to which the homotopy maps with degree 1 . Thus, $P_{S}^{n} \subseteq H_{S} \cup\left(N_{S}\right)_{\leq \eta} \subseteq\left(N_{S}\right)_{\leq t_{S}} \cup W_{S}(\xi)$.

Mapping down to $N$, we get maps $\psi_{S}: S \rightarrow N$, homotopic to $\omega^{n} \mid S$. Combining the maps $\psi_{S}$ for each such component $S$, we get a map $\psi: \partial_{0} M \rightarrow N$. We extend this to a piecewise straight map $\psi: M \rightarrow N \cup \partial^{\mathrm{c}} N$, sending each toroidal boundary component to a component of $\partial^{\mathrm{c}} N$. This map is homotopic to $\omega^{n}$. As before, we set $Q$ to be the set of points of $N$ to which $\psi$ maps with degree 1 . Thus the volume of $Q$ is bounded above in terms of $\tau(M)$.

Let $P^{n} \subseteq N$ be the set of points homologically between $\psi(\partial M)$ and $\omega^{n}(\partial M)$. Thus $R^{n}=Q \cup P^{n}$. Also, $P^{n} \subseteq N_{\leq t_{S}} \cup W(\xi)$. It follows that $R^{n} \subseteq N_{\leq t} \cup W(\xi)$, where $t$ is the maximum of $t_{0}$ and $t_{S}$ as $S$ ranges over the components of $\partial_{0} M$. Now $N \backslash N_{\leq \eta}^{\text {nc }} \subseteq N_{\leq \eta} \subseteq N_{\leq t}$ assuming $t \geq \eta$. Since $H \cap N_{\leq \eta}^{\text {nc }} \subseteq \bigcup_{n} R^{n}$, it follows that $H \subseteq \bar{N}_{\leq t} \cup W(\xi)$ as required.

To complete the proof, we need to explain how the product and compression body cases are adapted from Sections 3 and 4 respectively.

First suppose that $M \cong \Sigma \times[-1,1]$ and write $\partial_{0} M=\partial^{+} M \sqcup \partial^{+} M$, where $\partial^{ \pm} M \equiv \Sigma \times\{ \pm 1\}$. Let $\pi \subseteq \partial M$ be the multicurve corresponding to the set of $\mathbb{Z}$-cusps of $N$. (There are no $\mathbb{Z} \oplus \mathbb{Z}$-cusps in this case.) We construct a sequence of maps $\omega^{n}: \partial M \rightarrow N \cup \partial^{\mathrm{c}} N$ as in the general case above. Thus, $\omega^{n}$ is a nodal surface based on $\pi$, and each complementary surface $F$ is sent either to a component of $\partial H$ (when $F \in \mathscr{F}_{F}$ ), or to a singular hyperbolic surface $\omega^{n}(F)$, realising a complete multicurve $\gamma_{F}^{n}$ in a simply degenerate geometric end (when $F \in \mathcal{F}_{D}$ ). If $F \in \mathscr{F}_{F}$, we let $\gamma_{F}^{n}=\gamma_{F}$ be a complete multicurve in $F$ as given by Bers's Lemma. We set $\gamma^{n}=\pi \cup \bigcup_{F \in \mathcal{F}} \gamma_{F}^{n}$. Then $\gamma^{n} \supseteq \pi$ is a complete multicurve in $\partial M$. Let $\gamma^{n \pm}=\gamma^{n} \cap \partial^{ \pm} M$. By Lemma 2.4, we can connect $\gamma^{n-}$ to $\gamma^{n+}$ by a path in the pants graph. Realising these by singular surfaces as in Section 2, we construct a homotopy from $\omega^{n} \mid \partial^{-} M$ to $\omega^{n} \mid \partial^{+} M$. We can view this as a map $\psi^{n}: M \rightarrow N \cup \partial^{\mathrm{c}} N$, with $\psi^{n}\left|\partial M=\omega^{n}\right| \partial M$, and with $\psi^{n}(M) \subseteq N_{\leq t}$ where $t$ depends only on genus $(\Sigma)$. (As before, $W(\xi)=\emptyset$ in this case.) Now $H \subseteq \bigcup_{n} \psi^{n}(M)$, and so $H \subseteq N_{\leq t}$ as required.

Finally, we consider the case where $M$ is a compression body. Let $\partial^{+} M \subseteq \partial_{0} M$ be the outer boundary component, and let $\partial^{-} M$ be the inner boundary. This time, $\partial^{-} M$ may contain tori, namely the components of $\partial^{-} M \backslash \partial_{0} M$.

As before, we construct a sequence of nodal surfaces, $\omega^{n}: \partial^{+} M \rightarrow N \cup \partial^{c} N$. We can assume that there is no short compression in the simply degenerate parts. We follow the argument of Section 4 to construct a compression to a nodal multisurface homotopic to the inner boundary, and thence (via the product case) to another nodal multisurface, $\omega^{n}: \partial^{-} M \rightarrow N \cup \partial^{\mathrm{c}} N$, where $\omega^{n} \mid \partial^{-} M \cap \partial_{0} M$ maps each comple- 
mentary surface, $F$, either to a component of $\partial H$ or to a singular surface, $\omega^{n}(F)$, in a simply degenerate part, and where $\omega^{n}(F) \cap N_{\leq \eta}^{\text {nc }}$ goes out the corresponding end as $n \rightarrow \infty$. Also $\omega^{n} \mid \partial^{-} M \backslash \partial_{0} M$ maps each component homeomorphically to a toroidal component of $\partial^{c} N$. Once we have done this, we can conclude that $H \subseteq N_{\leq t} \cup W(\xi)$, where $t$ depends only on $\xi$ and genus $\left(\partial^{+} M\right)$.

There are a couple of complications we have to consider in the previous paragraph. Certain curves in our sequence of multicurves may get mapped into $\partial^{\mathrm{c}} N$ rather than to a closed geodesic. In this case, we just use a nodal surface to realise it. Also, after performing a compression, we may end up with an essential torus inner boundary component. These all correspond to $\mathbb{Z} \oplus \mathbb{Z}$-cusps, and can be homotoped to the corresponding torus component of $\partial^{\mathrm{c}} N$ by a homotopy in a bounded neighbourhood of $N_{\leq \eta}$.

\section{Convergence of limit sets}

The following is a consequence of Theorem 1.1, together with a result of McMullen [M]. I thank Al Marden for pointing this out to me. Special cases of the result were known before - where McMullen's hypotheses had been verified (cf. [E]).

Theorem 8.1. Suppose that $\left(G_{n}\right)_{n \in \mathbb{N}}$ is a sequence of isomorphic finitely generated groups acting properly discontinuously on $\mathbb{H}^{3}$. Suppose that $\left(G_{n}\right)$ is algebraically convergent and that $\left(G_{n}\right)$ also converges geometrically to a group $G$ acting properly discontinuously on $\mathbb{H}^{3}$. Then the limit sets $\Lambda G_{n}$ converge to the limit set $\Lambda G$ in the Hausdorff topology in $\partial \mathbb{H}^{3}$.

The definitions of algebraic and geometric convergence can be found in $[\mathrm{M}]$, for example. Briefly, algebraic convergence means that there are representations, $\rho_{n}$ and $\rho$, of a fixed finitely generated group $\Gamma$ into the isometry group of $\mathbb{H}^{3}$, with $G_{n}=\rho_{n}(\Gamma)$, so that $\rho_{n}(g)$ converges to $\rho(g)$ for all $g \in \Gamma$. All we will need from this is that, given any $x \in \mathbb{H}^{3}$, there is some $k \geq 0$ such that for all $n \in \mathbb{N}$, there is a finite generating set $S_{n}$ of $G_{n}$ such that $d(x, g x) \leq k$ for all $g \in S_{n}$. A sequence of groups $\left(G_{n}\right)$ converges geometrically to a group $G$ if every element of $G$ is the limit of a sequence of elements, $h_{n}$ of $G_{n}$, and moreover, if $h$ is any limit of a convergent sequence, $h_{n_{i}} \in G_{n_{i}}$ in any subsequence $\left(G_{n_{i}}\right)$ of $\left(G_{n}\right)$ then $h \in G$. (If $\left(G_{n}\right)_{n}$ also converges algebraically, as above, then one can see easily that $\rho(\Gamma) \subseteq G$, though these groups are not necessarily equal.)

Proof. By Selberg's lemma, after passing to a finite index subgroup, we can assume that the groups, $G_{n}$ are all torsion free. We write $H_{n}$ for the convex core of $N_{n}=$ $\mathbb{H}^{3} / G_{n}$. There are only finitely many possibilities for the homeomorphism type of $N_{n}$, so the topological complexity featuring in the statement of Theorem 1.1 is bounded. By Proposition 2.4 of [M], it is enough to show that there is some $t \geq 0$ 
such that for all $n$ each point of $H_{n}$ lies in some essential loop of length at most $t$. (Only the geometric convergence is needed to apply [M].) Thus, by Theorem 1.1 of this paper, it is enough to show that there is some $\xi>0$ such that for all $n$ there are no $\xi$-handles in $H_{n}$.

For this, we use algebraic convergence. Translating the earlier observation to $N_{n}$, this means there is some point $x_{n} \in N_{n}$ and a finite collection of loops based at $x_{n}$ of bounded length which generate $G_{n} \equiv \pi_{1}\left(N_{n}\right) \equiv \pi_{1}\left(H_{n}\right)$. There is no loss in taking $x_{n}$ and all the loops to lie in $H_{n}$. In this case, each compressing disc of $H_{n}$ must intersect at least one such loop essentially. This places a bound on the lengths $\xi$-handles, so if we take $\xi$ small enough, we can assume there are none, and the result follows.

\section{References}

[A] I. Agol, Tameness and hyperbolic 3-manifolds. Preprint 2004. arXiv:math/0405568

[Be] L. Bers, An inequality for Riemann surfaces. In Differential geometry and complex analysis, Springer-Verlag, Berlin 1985, 87-93. Zbl 0575.30039 MR 780038

[Bi] B. Bielefeld (ed.), Conformal dynamics problem list. Preprint 1990. arXiv:math/9201271

[Bo] F. Bonahon, Bouts des variétés hyperboliques de dimension 3. Ann. of Math. (2) 124 (1986), 71-158. Zbl 0671.57008 MR 847953

[BrMNS] J. Brock, Y. N. Minsky, H. Namazi, and J. Souto, Splittings, models and bounds in hyperbolic geometry. In preparation.

[BrS] J. Brock and J. Souto, Volume and distance in the pants complex. In preparation.

[CalG] D. Calegari and D. Gabai, Shrinkwrapping and the taming of hyperbolic 3manifolds. J. Amer. Math. Soc. 19 (2006), 385-446. Zbl 1090.57010 MR 2188131

[Can] R. D. Canary, A covering theorem for hyperbolic 3-manifolds and its applications. Topology 35 (1996), 751-778. Zbl 0863.57010 MR 1396777

[E] R. A. Evans, Uniformly bounded radii of balls in convex cores of hyperbolic 3manifolds. Preprint 2005.

[F1] C. E. Fan, Injectivity radius bounds in $I$-bundle hyperbolic convex cores. Preprint 1999. arXiv:math/9907052

[F2] C. E. Fan, Injectivity radius bounds in hyperbolic convex cores I. Preprint 1999. arXiv:math/9907058

[HT] A. Hatcher and W. Thurston, A presentation for the mapping class group of a closed orientable surface. Topology 19 (1980), 221-237. Zbl 0447.57005 MR 579573

[J] K. Johannson, Homotopy equivalences of 3-manifolds with boundaries. Lecture Notes in Math. 761, Springer-Verlag, Berlin 1979. Zbl 0412.57007 MR 551744

[M] C. T. McMullen, Renormalization and 3-manifolds which fiber over the circle. Ann. of Math. Stud. 142, Princeton University Press, Princeton, NJ, 1996. Zbl 0860.58002 MR 1401347 
[S] G. A. Swarup, Two finiteness properties in 3-manifolds. Bull. London Math. Soc. 12 (1980), 296-302. Zbl 0457.57007 MR 576979

Received October 08, 2010; revised August 11, 2011

B. H. Bowditch, Mathematics Institute, University of Warwick, Coventry, CV4 7AL, Great Britain

E-mail: B.H.Bowditch@warwick.ac.uk 